\author{
Ecología
}

\title{
Abundancia y distribución de ácaros parásitos (Eutrombicula araucanensis y Pterygosoma sp.) en lagartijas (Liolaemus pictus) de Chile central
}

\section{Abundance and distribution of parasitic mites (Eutrombicula araucanensis and Pterygosoma sp.) on lizards (Liolaemus pictus) of central Chile}

\author{
Mario Espinoza-Carniglia ${ }^{a}$, Amado Pérez-Leiva ${ }^{a}$, María Carolina Silva-de la Fuente ${ }^{b}$, \\ Pedro Victoriano-Sepúlveda ${ }^{a}$ y Lucila Moreno-Salas ${ }^{a}, *$ \\ ${ }^{a}$ Departamento de Zoología, Facultad de Ciencias Naturales y Oceanográficas, Universidad de Concepción. Barrio Universitario s/n, Casilla 160C, \\ Concepción, Chile \\ ${ }^{\mathrm{b}}$ Departamento de Ciencias Pecuarias, Facultad de Ciencias Veterinarias, Universidad de Concepción. Avenida Vicente Méndez, 595, Chillán, Chile
}

Recibido el 25 de marzo de 2015; aceptado el 28 de agosto de 2015

Disponible en Internet el 12 de febrero de 2016

\begin{abstract}
Resumen
Con el objetivo de analizar la abundancia y distribución de ácaros en la lagartija Liolaemus pictus y su relación con el tamaño corporal y sexo del hospedador, se extrajeron 835 ácaros de 134 individuos de L. pictus capturados en Chile central —regiones de la Araucanía hasta Los Lagos, incluyendo la isla de Chiloé-. Se calculó la prevalencia y abundancia total de ácaros por hospedador y abundancia por zona corporal. Se analizó la relación entre el tamaño corporal del hospedador y la abundancia de ácaros. El 39\% de las lagartijas presentó ácaros, identificándose las especies Eutrombicula araucanensis (prevalencia 34.3\%) y Pterygosoma sp. (prevalencia 8.2\%). No se encontró asociación significativa entre tamaño corporal del hospedador ni el sexo con la abundancia de ácaros. Las zonas corporales del hospedador mayormente parasitadas fueron la femoral y flanco, aunque la zona femoral estuvo parasitada solamente por E. araucanensis. Se plantea que la forma de las escamas puede ser el factor que esté influenciando directamente la distribución corporal de estas especies de ácaros en L. pictus en Chile.

Derechos Reservados (C) 2015 Universidad Nacional Autónoma de México, Instituto de Biología. Este es un artículo de acceso abierto distribuido bajo los términos de la Licencia Creative Commons CC BY-NC-ND 4.0.
\end{abstract}

Palabras clave: Trombiculidae; Pterygosomatidae; Infestación; Prevalencia

\begin{abstract}
In order to analyze the abundance and distribution of mites in the lizard Liolaemus pictus and its relationship with the body size and sex of the host, 835 mites from 134 specimens of L. pictus captured in central Chile —Araucanía Region to Los Lagos, including Chiloé Island- were studied. Prevalence, total abundance and abundance for each body region of the host were calculated. The relationship between the host body size and abundance of mites was analyzed. Mites were found in 39\% of lizards, identifying 2 species Eutrombicula araucanensis (prevalence 34.3\%) and Pterygosoma sp. (prevalence 8.2\%). No significant association between host body size, sex and abundance of mites was found. The host body areas that had higher abundance of mites were: femoral and flank, although the femoral area was only parasitized by E. araucanensis. We propose that the shape of the lizard scales could be the factor that is directly influencing the body distribution of these species of mites in L. pictus in Chile. All Rights Reserved @ 2015 Universidad Nacional Autónoma de México, Instituto de Biología. This is an open access item distributed under the Creative Commons CC License BY-NC-ND 4.0.
\end{abstract}

Keywords: Trombiculidae; Pterygosomatidae; Infestation; Prevalence

\footnotetext{
* Autora para correspondencia.

Correo electrónico: 1umoreno@udec.cl (L. Moreno-Salas).

La revisión por pares es responsabilidad de la Universidad Nacional Autónoma de México.
} 


\section{Introducción}

El mayor tamaño corporal de un hospedador provee mayor superficie disponible, lo que permite la adhesión de una mayor cantidad de parásitos (Krasnov, Poulin y Morand, 2006; Morand y Guégan, 2000). Esta relación ha sido encontrada en organismos endoparásitos (Grutter y Poulin, 1998; Morand y Guégan, 2000; Poulin y Rohde, 1997) y en ectoparásitos (Peña, Contreras y Castañeda, 2004; Rubio y Simonetti, 2009; Schall, Prendeville y Hanley, 2000). A pesar de esto, el tamaño corporal del hospedador y el nivel de infestación no siempre están relacionados (Cunha-Barros y Rocha, 2000; Cunha-Barros et al., 2003), probablemente porque los ectoparásitos también están influenciados por factores ambientales como humedad y radiación solar (Clopton y Gold, 1993).

El sexo del hospedador es otro factor que se ha puesto a prueba para tratar de explicar la abundancia de parásitos en un hospedador. Estudios realizados en diversos taxones (e.g., salamandras, pitón bola, lagartijas, roedores y aves) muestran que los hospedadores macho tienden a presentar mayor carga parasitaria que las hembras (Anthony, Mendelson y Simons, 1994; Aubret, Bonnet, Harris y Maumelat, 2005; Klukowski y Nelson, 2001; Moore y Wilson, 2002; Morand, Goüy de Bellocq, Stanko y Miklisova, 2004; Poulin, 1996; Salkeld y Schwarzkopf, 2005; Schall y Marghoob, 1995; Schall et al., 2000; Tschirren, Fitze y Richner, 2003; Zuk, 1990). Se ha propuesto que estas diferencias en cargas parasitarias entre sexos pueden ocurrir por factores morfológicos — tamaño del hospedador-, factores fisiológicos — diferencias en los niveles hormonales o condiciones reproductoras- y factores ecológicos — comportamiento, interacciones sociales y alimentación(Klukowski y Nelson, 2001; Marler y Moore, 1989; Poulin, 1996).

A nivel de infrapoblaciones — poblaciones de parásitos presentes en un individuo hospedador en un tiempo particular-, los parásitos tienden a distribuirse agrupadamente en algunos sectores del cuerpo de sus hospedadores por mayor disponibilidad de alimento o refugio. Por ejemplo, en lagartijas, los ácaros se encuentran principalmente en la zona gular, e.g., bolsillos de ácaros (Arnold, 1986; García-de la Peña, Gadsden y SalasWestphal, 2010), donde la piel es más elástica y tiende a sanar rápidamente, por el mayor nivel de irrigación sanguínea, minimizando el área dañada del cuerpo de su hospedador (García-de la Peña et al., 2010).

La lagartija Liolaemus pictus Duméril y Bibron, 1837, es una especie perteneciente a la familia Liolaemidae (Etheridge y Espinoza, 2000) que se caracteriza por habitar zonas templadas y frías (Kubisch, Fernández e Ibargüengoytía, 2011), encontrándose en Argentina en la provincia de Río Negro, en Chile entre Vilches Alto $\left(35^{\circ} 35^{\prime} \mathrm{S}, 71^{\circ} 05^{\prime} \mathrm{O}\right)$ y la Reserva Nacional Futaleufú $\left(43^{\circ} 15^{\prime} \mathrm{S}, 71^{\circ} 47^{\prime} \mathrm{O}\right)$, habitando también en la isla Chiloé e isla Mocha (Elgueta et al., 2006; Pincheira-Donoso y Núñez, 2005; Veloso y Navarro, 1988). Su alimentación es insectívora y frugívora, siendo un dispersor de semillas de plantas y arbustos (Willson, Sabag, Figueroa, Armesto y Caviedes, 1996). Es una lagartija de hábitos diurnos y su modo reproductor es vivíparo, teniendo una frecuencia bianual o trianual (Ibargüengoytia y Cussac, 1996). Actualmente se encuentra categorizada como vulnerable (Elgueta et al., 2006). Para L. pictus se han descrito 6 especies de ácaros parásitos: 2 trombicúlidos -Eutrombicula araucanensis Stekol'nikov y González-Acuña, 2010 y Morelacarus sp. Stekol' nikov y González-Acuña, 2015-, parásitos solo en estado larval, con ninfas y adultos de vida libre (Hoffmann, 1990; Wharton y Fuller, 1952), y 4 pterigosómidos Pterygosoma ligare, $P$. formosus, $P$. ovata y P. levissima Fajfer y GonzálezAcuña, 2013, descritos como parásitos permanentes (Fajfer, 2012).

En Chile, a pesar de los antecedentes históricos que muestran la recurrente presencia de ácaros en varias especies de reptiles, existe escasa información de su ecología. El objetivo de este estudio es determinar la distribución corporal y abundancia de ácaros en $L$. pictus, identificando las zonas corporales mayormente parasitadas por ácaros. Además, se evalúa la relación del tamaño corporal y sexo de L. pictus con respecto a la abundancia de ácaros.

\section{Materiales y métodos}

Se recolectaron y analizaron 134 individuos de L. pictus: 58 machos, 50 hembras y 26 juveniles, obtenidos desde la región de la Araucanía ( $37^{\circ} 53^{\prime} 40.2^{\prime}$ S, $72^{\circ} 28^{\prime} 29^{\prime}$ ” O) hasta la región de Los Lagos ( $\left.43^{\circ} 53^{\prime} 00.8^{\prime \prime} \mathrm{S}, 72^{\circ} 55^{\prime} 45.3^{\prime \prime} \mathrm{O}\right)$ en Chile, incluyendo la isla de Chiloé (fig. 1), abarcando la distribución actual descrita para L. pictus (Vera-Escalona, Coronado, Muñoz-Mendoza y Victoriano, 2010) (tabla 1). Los ejemplares de L. pictus fueron capturados a mano y con lazo, registrándose el sitio de captura, tamaño, sexo —macho $(\mathrm{M})$ o hembra $(\mathrm{H})$ - y estado de desarrollo — juvenil (J) o adulto (A)—. Para diferenciar estado adulto de juvenil se consideró la presencia de caracteres sexuales completamente desarrollados, basándose en el criterio de la hembra grávida de menor tamaño $(46.59 \mathrm{~mm})$ y la presencia de hemipenes y poros cloacales, en el caso de los machos (Mella, 2005). Todos los individuos fueron sacrificados y conservados en alcohol al 95\%. Posteriormente, cada ejemplar de L. pictus fue observado bajo estereomicroscopio y se recolectaron los ácaros de todo el cuerpo del hospedador, considerando 12 zonas corporales (fig. 2). Los ácaros recolectados fueron preservados en alcohol al 95\%; aclarados en solución Nesbitt y montados de forma permanente en solución Berlese (Krantz y Walter, 2009), posteriormente se identificaron con base en Brennan y Goff (1977), Fajfer y González-Acuña (2013), Krantz y Walter (2009) y Stekol'nikov y González-Acuña (2010), bajo un microscopio óptico de luz (Motic modelo BA210) con aumento 400x. Los ejemplares montados fueron almacenados en la colección del laboratorio de Ecología Parasitaria, Universidad de Concepción.

Se calculó la abundancia total y frecuencia por zona del cuerpo del hospedador: zona anterior: mandibular [Ma], gular [Gu], auditiva [Au; lado izquierdo y derecho], antehumeral [Ah; lado izquierdo y derecho], axilar [Ax; lado izquierdo y derecho] y abdominal [Ab]; zona del flanco [Fl; lado izquierdo y derecho]; zona femoral [Fe; lado izquierdo y derecho]; zona posterior: inguinal [In; lado izquierdo y derecho], cloacal [Cl], base de la cola [Co] y línea dorsal [Ld] (fig. 2). Previo a los análisis, se diferenció el lado izquierdo y derecho con la prueba 
Tabla 1

Localidades de muestreo de Liolaemus pictus en Chile central. Se indican las coordenadas de los sitios de muestreo y el número de lagartijas analizadas.

\begin{tabular}{|c|c|c|c|c|}
\hline Fecha de muestreo & Localidad & Latitud (S) & Longitud $(\mathrm{O})$ & Número de individuos \\
\hline 06-03-2007 & Curacautín & $38^{\circ} 13^{\prime} 04.9^{\prime \prime}$ & $71^{\circ} 48^{\prime} 34.2^{\prime \prime}$ & 7 \\
\hline 08-03-2007 & Parque Nacional Villarrica & $39^{\circ} 25^{\prime} 11.0^{\prime \prime}$ & $71^{\circ} 46^{\prime} 50.7^{\prime \prime}$ & 3 \\
\hline 09-03-2007 & San Juan de la Costa & $40^{\circ} 34^{\prime} 30.1^{\prime \prime}$ & $73^{\circ} 42^{\prime} 39.6^{\prime \prime}$ & 5 \\
\hline $10-03-2007$ & Puyehue & $40^{\circ} 46^{\prime} 59.5^{\prime \prime}$ & $72^{\circ} 13^{\prime} 00.1^{\prime \prime}$ & 7 \\
\hline $11-03-2007$ & Puerto Montt, Camino Alerce Andino & $41^{\circ} 36^{\prime} 15.2^{\prime \prime}$ & $72^{\circ} 38^{\prime} 51.2^{\prime}$ & 10 \\
\hline $12-03-2007$ & Ancud, Chiloé & $42^{\circ} 06^{\prime} 57.0^{\prime \prime}$ & $73^{\circ} 49^{\prime} 10.3^{\prime \prime}$ & 10 \\
\hline $13-03-2007$ & La Chacra, Chiloé & $42^{\circ} 23^{\prime} 05.6^{\prime \prime}$ & $73^{\circ} 54^{\prime} 47.1^{\prime \prime}$ & 4 \\
\hline $13-03-2007$ & Chonchi, Chiloé & $42^{\circ} 37^{\prime} 30.9^{\prime \prime}$ & $74^{\circ} 06^{\prime} 28.9^{\prime \prime}$ & 3 \\
\hline $15-03-2007$ & Chaitén & $42^{\circ} 59^{\prime} 30.4^{\prime \prime}$ & $72^{\circ} 26^{\prime} 03.8^{\prime \prime}$ & 8 \\
\hline 22-03-2007 & Cisnes & $43^{\circ} 53^{\prime} 00.8^{\prime \prime}$ & $72^{\circ} 55^{\prime} 45.3^{\prime \prime}$ & 1 \\
\hline $11-01-2008$ & Cuenca Río Puelo & $41^{\circ} 39^{\prime} 00.0^{\prime \prime}$ & $72^{\circ} 16^{\prime} 00.0^{\prime \prime}$ & 2 \\
\hline $19-01-2008$ & Salto el Indio, Malalcahuello & $38^{\circ} 27^{\prime} 42.5^{\prime \prime}$ & $71^{\circ} 44^{\prime} 31.5^{\prime \prime}$ & 4 \\
\hline $22-01-2008$ & Camino Parque Oncol & $39^{\circ} 44^{\prime} 52.3^{\prime \prime}$ & $73^{\circ} 20^{\prime} 54.5^{\prime \prime}$ & 5 \\
\hline $23-01-2008$ & Tres Chiflones Altos, Reserva Valdivia & $40^{\circ} 04^{\prime} 08.1^{\prime \prime}$ & $73^{\circ} 14^{\prime} 53.2^{\prime \prime}$ & 5 \\
\hline $25-01-2008$ & Entrada a Parque Tantauco, Chiloé & $43^{\circ} 02^{\prime} 57.8^{\prime \prime}$ & $73^{\circ} 52^{\prime} 38.5^{\prime \prime}$ & 7 \\
\hline 03-12-2008 & Camino Hueicolla, Alerce Costero & $40^{\circ} 10^{\prime} 09.0^{\prime \prime}$ & $73^{\circ} 27^{\prime} 47.0^{\prime \prime}$ & 8 \\
\hline $03-12-2008$ & Camino La Unión, Hueicolla & $40^{\circ} 16^{\prime} 06.0^{\prime \prime}$ & $73^{\circ} 19^{\prime} 43.0^{\prime \prime}$ & 5 \\
\hline 05-04-2009 & Valle Las Trancas, camino a Shangrilá & $37^{\circ} 53^{\prime} 40.2^{\prime \prime}$ & $72^{\circ} 28^{\prime} 29.0^{\prime \prime}$ & 4 \\
\hline $25-11-2010$ & Panguipulli, volcán Choshuenco & $39^{\circ} 55^{\prime} 04.6^{\prime \prime}$ & $71^{\circ} 58^{\prime} 53.0^{\prime \prime}$ & 3 \\
\hline $10-11-2011$ & Reserva Nacional Llanquihue & $41^{\circ} 19^{\prime} 38.4^{\prime \prime}$ & $72^{\circ} 25^{\prime} 24.9^{\prime \prime}$ & 9 \\
\hline $11-11-2011$ & Faldas volcán Osorno & $41^{\circ} 07^{\prime} 58.9^{\prime \prime}$ & $72^{\circ} 32^{\prime} 07.5^{\prime \prime}$ & 12 \\
\hline $12-11-2011$ & Parque Nacional Puyehue & $40^{\circ} 40^{\prime} 55.8^{\prime \prime}$ & $72^{\circ} 01^{\prime} 17.0^{\prime \prime}$ & 12 \\
\hline
\end{tabular}

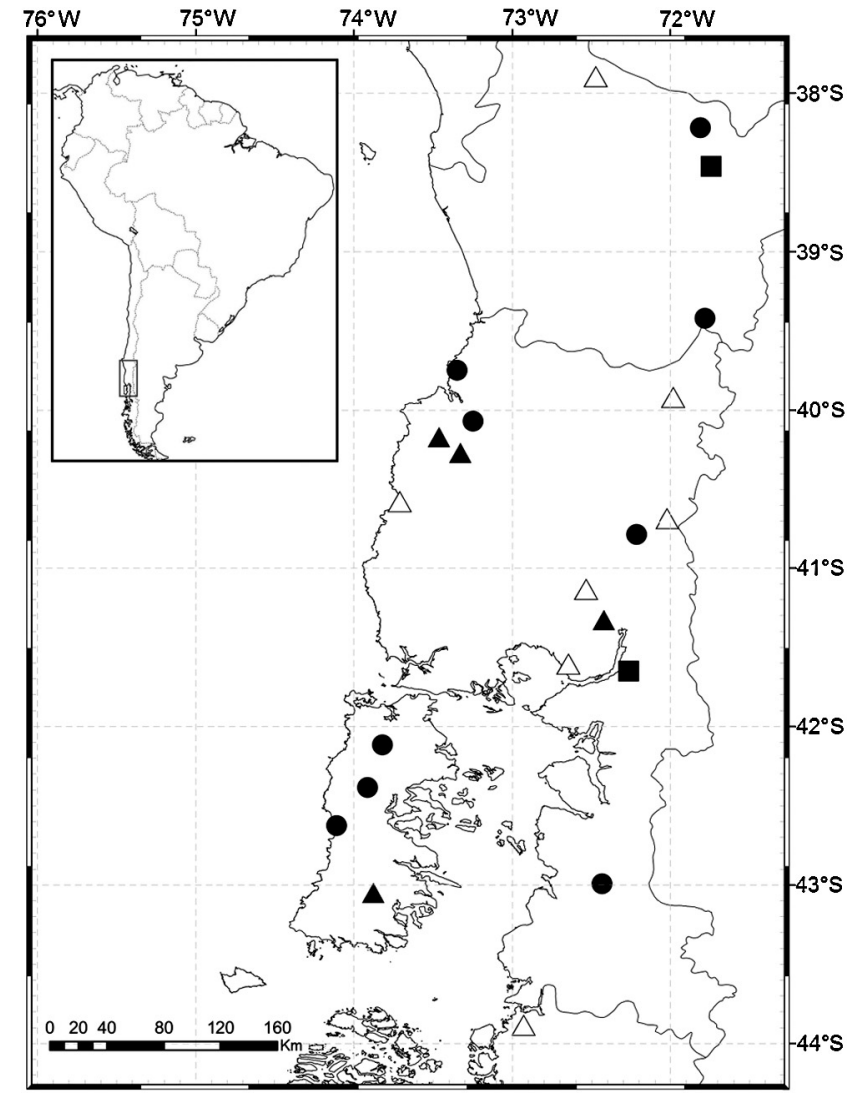

Figura 1. Localización geográfica de los puntos de muestreo de Liolaemus pictus. Triángulos blancos: localidades sin ácaros parásitos; triángulos negros: localidades con presencia de Eutrombicula araucanensis y Pterygosoma sp.; círculos negros: localidades solo con E. araucanensis y cuadrados negros: localidades solo con Pterygosoma sp. de Wilcoxon, no observándose diferencias significativas entre ambos lados, por lo que los datos fueron evaluados en conjunto para cada zona $(n=134, p>0.05, \mathrm{gl}=133)$. Se calculó la prevalencia —número de hospedadores infestados dividido entre el total de hospedadores examinados, $\mathrm{P} \%$-; abundancia promedio —número de parásitos por hospedador dividido por el total de hospedadores, incluyendo hospedadores no infestados, AP-; intensidad promedio — número total de parásitos dividido entre el número de hospedadores parasitados, IP-. Todos estos registros fueron realizados por género (hembra o macho) y estado de desarrollo (adulto o juvenil).

Se evaluó la normalidad de los datos mediante la prueba de Shapiro-Wilk (Shapiro y Wilk, 1965), y como estos no se ajustaron a la distribución normal $(n=134, p<0.05, \mathrm{gl}=133)$ se utilizó estadística no paramétrica. Para evaluar las diferencias en abundancias e intensidades parasitarias entre machos, hembras y juveniles se utilizó la prueba de Wilcoxon. Esta misma prueba fue realizada para evaluar las diferencias de LHC entre machos y hembras (Wilcoxon, 1945). Para evaluar las diferencias en la distribución de los ácaros en el cuerpo del hospedador se utilizó la prueba de Kruskal-Wallis y la de signos de rangos de Wilcoxon. Para evaluar diferencias en la prevalencia de cada especie de ácaro en cada lagartija analizada se realizó una prueba de Chi-cuadrado; esta misma prueba fue realizada para evaluar las diferencias entre prevalencia entre machos y hembras, juveniles y adultos. Se aplicó correlación de Spearman para evaluar la asociación entre tamaño corporal del hospedador (LHC) y abundancia de ácaros. Para todos los análisis estadísticos se utilizaron los programas Quantitative Parasitology (Reiczigel y Rózsa, 2005), InfoStat (Di Rienzo et al., 2015) y JMP® 7.0.1 (SAS Institute Inc). El nivel de significación para todas las pruebas estadísticas realizadas fue de 0.05 . 

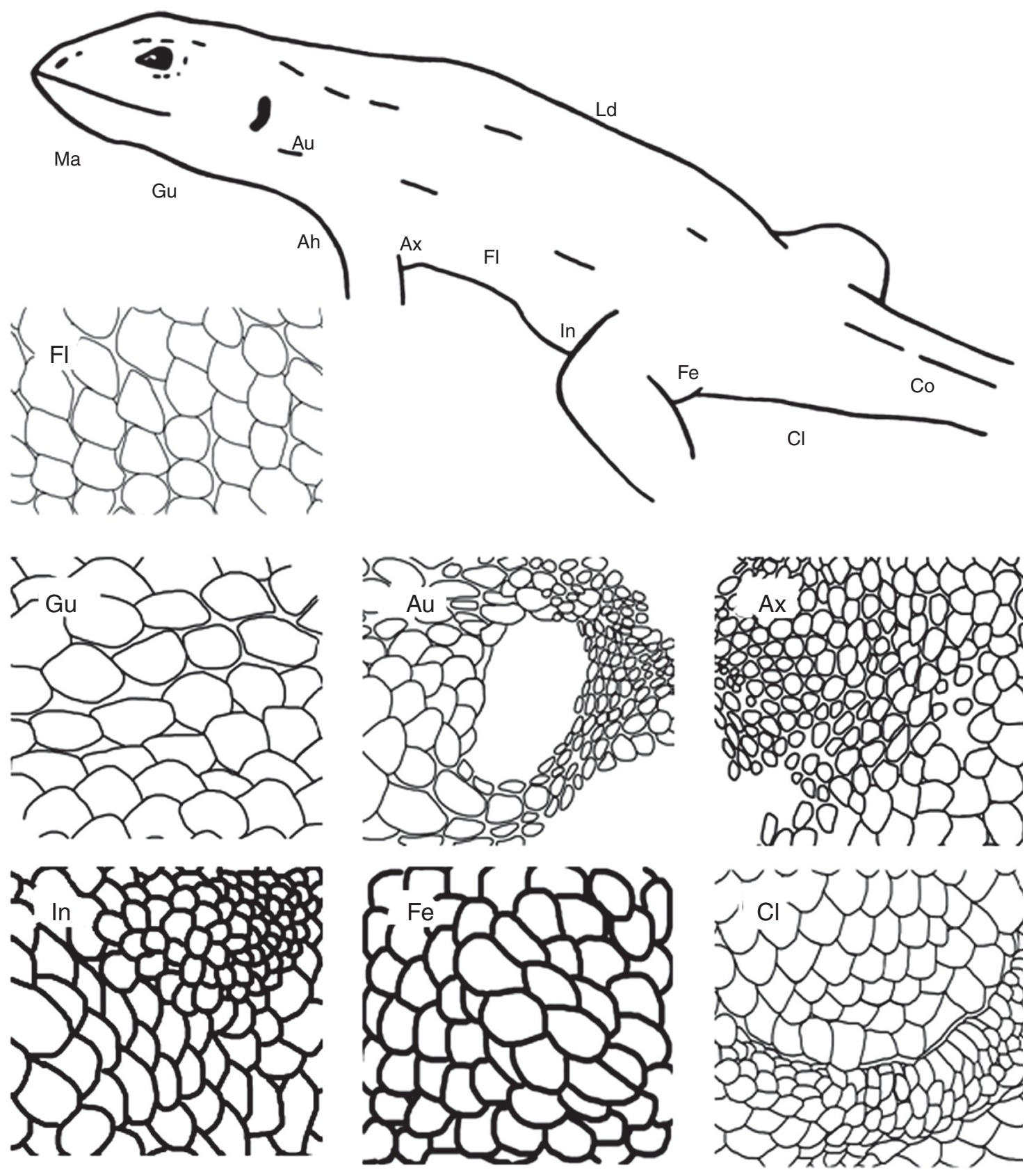

Figura 2. Zonas corporales de Liolaemus pictus consideradas en este estudio. Las imágenes muestran las escamas de algunas zonas corporales.

\section{Resultados}

Del total de individuos L. pictus analizados, $52(38.8 \%)$ se encontraron infestados por ácaros, aislándose en total 835 ácaros; el 15.6\% perteneció al género Pterygosoma (Acari: Pterygosomatidae) y el $84.4 \%$ correspondió a E. araucanensis. Esta última especie presentó una prevalencia del $34.3 \%$ y abundancia promedio de 5.26 ácaros por hospedador, valores que son significativamente mayores a los presentados por Pterygosoma sp., cuya prevalencia y abundancia promedio fueron del $8.2 \mathrm{y}$ $0.97 \%$ ácaros por individuo, respectivamente (tabla 2).

No encontramos diferencias significativas entre las LHC de machos $(n=58$, promedio $=58.18 \mathrm{~cm}, \mathrm{DE} \pm 0.96) \mathrm{y}$ hembras $(\mathrm{n}=50$, promedio $=57.85 \mathrm{~cm}, \mathrm{DE} \pm 1.03)(p=0.7510$; $\mathrm{gl}=107)$. Los juveniles de L. pictus se encontraron menos parasitados que los adultos (AP: $p=0.037, \mathrm{P} \%: p=0.024, \mathrm{gl}=$ 133; tabla 3). Las lagartijas hembras no presentaron diferencias en niveles de prevalencia (M: $n=58 ; \mathrm{H}: n=50 ; \mathrm{P} \%$ : $p=0.693, \mathrm{gl}=107)$, intensidad promedio (M: $n=58 ; \mathrm{H}: n=50$; IP: $p=0.694, \mathrm{gl}=107)$ y abundancia promedio (M: $n=58 ; \mathrm{H}$ : $n=50 ;$ AP: $p=0.527, \mathrm{gl}=107)$ con respecto a los machos para E. araucanensis. Para Pterygosoma sp. tampoco se encontró diferencia en la abundancia de ácaros (M: $n=58 ; \mathrm{H}: n=50$; $p=0.06, \mathrm{gl}=107$ ) entre hospedadores machos y hembras. Tampoco se observó asociación significativa entre la abundancia de ácaros y el tamaño del hospedador para $E$. araucanensis $(n=134, p=0.8221 ;$ rho $=0.02, \mathrm{gl}=133)$ y tampoco para Pterygosoma sp. $(n=134, p=0.5672 ;$ rho $=0.05, \mathrm{gl}=133)$. 
Tabla 2

Niveles de infestación por ácaros aislados sobre Liolaemus pictus en el presente estudio. En paréntesis se indica los intervalos de confianza.

\begin{tabular}{llll}
\hline Estadísticos descriptivos & E. araucanensis & Pterygosoma sp. & Total \\
\hline Hospedadores infestados & 46 & 11 & 52 \\
Prevalencia & $0.343(0.2659 ; 0.4289)$ & $0.082(0.0431 ; 0.1409)$ & $0.388(0.3092 ; 0.4738)$ \\
Intensidad promedio & $15.33(11.09 ; 21.74)$ & $11.82(6.55 ; 22.45)$ & $16.06(12.06 ; 21.90)$ \\
Abundancia promedio & $5.26(3.52 ; 7.95)$ & $0.97(0.40 ; 2.19)$ & $6.23(4.36 ; 9.01)$ \\
\hline
\end{tabular}

Tabla 3

Niveles de infestación por ácaros entre hembras, machos, juveniles y adultos de Liolaemus pictus. En paréntesis se indican los intervalos de confianza.

\begin{tabular}{lclll}
\hline & Número de hospedadores & Prevalencia & Intensidad promedio & Abundancia promedio \\
\hline Hembras $(\mathrm{H})$ & 50 & $0.360(0.2384 ; 0.5000)$ & $13.72(8.94 ; 20.89)$ & $4.94(2.72 ; 8.50)$ \\
Machos $(\mathrm{M})$ & 58 & $0.414(0.2919 ; 0.5433)$ & $15.96(9.46 ; 27.17)$ & $6.60(3.67 ; 12.41)$ \\
Juveniles $(\mathrm{J})$ & 26 & $0.154(0.0544 ; 0.3436)$ & $17.25(5.00 ; 33.75)$ & $2.65(0.54 ; 8.38)$ \\
Adultos $(\mathrm{H}+\mathrm{M})$ & 108 & $0.389(0.3004 ; 0.4860)$ & $15.00(10.55 ; 22.12)$ & $5.83(3.83 ; 9.00)$ \\
\hline
\end{tabular}

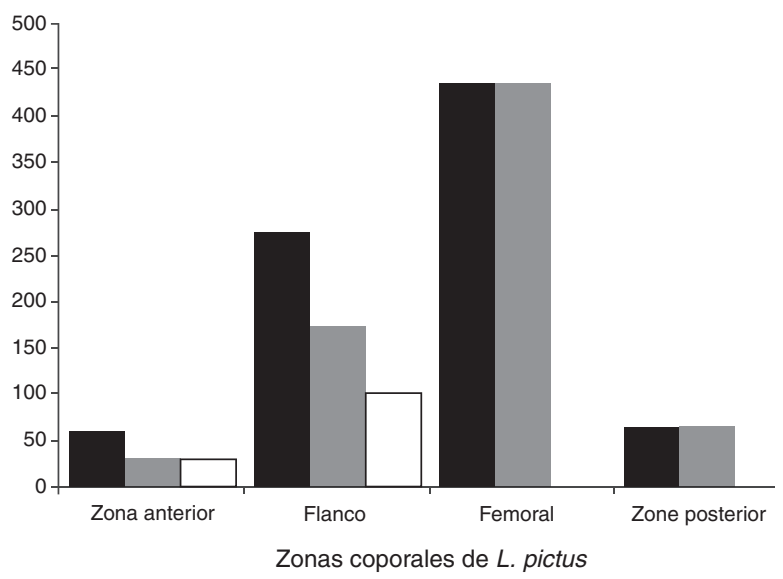

Figura 3. Abundancia de ácaros por zona corporal de Liolaemus pictus. Barras negras: total de ácaros, barras grises: Eutrombicula araucanensis y barras blancas: Pterygosoma sp.

En cuanto a la infestación observada en las zonas corporales del hospedador, el $61.7 \%$ de E. araucanensis se encontró en la zona femoral y el $24.7 \%$ se ubicó en el flanco; mientras que Pterygosoma sp. no mostró preferencia por alguna zona corporal $(n=134, p=0.1029, \mathrm{gl}=133)$, aunque el $76.9 \%$ se encontró en la zona del flanco (fig. 3). La zona inguinal y mandibular del hospedador no estaba parasitada por ninguna de las 2 especies de ácaros; mientras que en las zonas del flanco, gular y antehumeral coexisten ambas especies de ácaros.

\section{Discusión}

Se encontraron 2 especies de ácaros parásitos $E$. araucanensis y Pterygosoma sp. La primera especie ha sido descrita en estadio larval en L. pictus, Pristidactylus torquatus (Philippi, 1861), Liolaemus tenuis (Dumeril y Bibron, 1837) y Liolaemus lemniscatus Gravenhorst, 1838 (Stekol'nikov y González-Acuña, 2010, 2015); mientras que las especies del género Pterygosoma parasitan a lagartijas del género Liolaemus, además de especies de Agamidae y Gerrhosauridae (Fajfer, 2012). En este estudio, las abundancias y prevalencias documentadas en las 22 localidades fueron menores a las registradas por Rubio y Simonetti
(2009), quienes encuentran un 100\% de prevalencia en L. tenuis Duméril y Bibron, 1837. Por el contrario, Carothers y Jaksic (2001) hallan que L. tenuis alcanza el $49 \%$ de prevalencia de ácaros y esta prevalencia es variable entre distintas especies de hospedadores del género Liolaemus (Carothers y Jaksic, 2001). Sin embargo, es importante considerar que Carothers y Jaksic (2001) analizaron solamente una localidad para evaluar la distribución altitudinal y los niveles de prevalencia pueden ser bastante variables en un rango distribucional más amplio. Esta variación latitudinal en los niveles de parasitismo de E. araucanensis ha sido estudiada en el rango de distribución de L. pictus. Esta especie de ácaro presenta localidades de su distribución con el $100 \%$ de prevalencia, sin embargo, otras localidades tienen prevalencias mucho menores; probablemente las variaciones microclimáticas en los niveles de radiación solar, temperatura y humedad en el hábitat fragmentado del hospedador estén causando estas variaciones (Espinoza-Carniglia, Silva-de la Fuente, Pérez, Victoriano y Moreno, 2015). Otras especies de lagartijas de distintos géneros se presentan muy parasitadas, como es el caso de Tropidurus torquatus Wied-Neuwied, 1820, la cual presenta altos niveles de intensidad promedio (86.4 ácaros/hospedador), con respecto a Mabuya agilis Raddi, 1823 (20.9 ácaros/hospedador), M. macrorhyncha Hoge, 1946 (11.1 ácaros/hospedador) y Cnemidophorus littoralis Rocha, Araujo, Vrcibradic y Costa, 2000 (19.1 ácaros/hospedador) (CunhaBarros et al., 2003). Las especies de lagartijas anteriormente mencionadas presentaban prevalencias del $100 \%$, excepto $C$. littoralis con una prevalencia del 95.2\% (Cunha-Barros et al., 2003). La forma de las escamas y el comportamiento de las lagartijas serían factores determinantes de la abundancia de ácaros, por lo que la alta abundancia y prevalencia podría ser explicada por las escamas imbricadas y el comportamiento sedentario de los hospedadores (Cunha-Barros et al., 2003), lo que podría facilitar la adhesión de larvas. La menor abundancia y prevalencia documentada en el presente estudio, con respecto a otros autores, podría ser efecto de la fragmentación de los bosques templados (Rubio y Simonetti, 2009), ya que en cada parche se modifican las condiciones de temperatura, humedad e incidencia de la luz, factores determinantes en el ciclo de vida de los ácaros (Clopton y Gold, 1993). 
Contrario a lo esperado, no se observó asociación entre el tamaño de L. pictus (LHC) y la abundancia de ácaros, lo que contrasta con otros estudios que indican que existe estrecha relación entre estos parámetros (Tompkins y Clayton, 1999). Por lo tanto, el tamaño del cuerpo del hospedador parece no ser siempre un factor importante que afecta los niveles de infestación por ácaros (Cunha-Barros y Rocha, 1995; Cunha-Barros et al., 2003; Delfino, Ribeiro, Furtado, Anjos y Almeida, 2011; García-de la Peña et al., 2004; Heredia, Vicente, Robles y Halloy, 2014; Krasnov, Shenbrot, Khokhlova y Degen, 2004; Rocha et al., 2008), ya que otros factores podrían estar afectando la carga presente en el hospedador, como cambios hormonales (Klukowski y Nelson, 2001) o comportamiento (Poulin, 1996). Por ejemplo, los juveniles que son de menor tamaño se encontraron menos parasitados que los adultos, lo que podría ser debido a que los individuos juveniles experimentan mudas más constantemente que los adultos, dificultando el establecimiento de parásitos sobre su superficie corporal (Camacho y Pérez-Lara, 2009; García-de la Peña et al., 2010; Rubio y Simonetti, 2009).

Respecto al sexo, no se encontró diferencia significativa en la prevalencia y abundancia media entre machos y hembras; resultados similares han sido obtenidos en otros estudios (Delfino et al., 2011; García-de la Peña et al., 2004), explicando que en estos casos existe baja selectividad de los ácaros por alguno de los sexos (Carvalho, Araújo y da Silva, 2006). Estos resultados contrastan con otras investigaciones que han encontrado mayor cantidad de parasitismo para hospedadores machos (Rubio y Simonetti, 2009; Schall et al., 2000), explicado por los niveles de testosterona, ya que es una hormona que estimula la territorialidad, actividad locomotora y el tamaño del ámbito hogareño de lagartijas machos (DeNardo y Sinervo, 1994; Marler y Moore, 1989), aumentando la cantidad de hábitats visitados por los hospedadores, dejándolos expuestos a una mayor cantidad de larvas de ácaros que buscan hospedadores (Eisen, Eisen y Lane, 2001; Tälleklint-Eisen y Eisen, 1999). Esta hormona, además, ha sido asociada a un efecto inmunosupresor, lo que haría a los machos más susceptibles a la infestación, sin embargo, este efecto inmunosupresor no estaría asociado a una mayor carga parasitaria (Oppliger, Giorgi, Conelli, Nembrini y John-Alder, 2004).

Respecto a la distribución corporal, existen zonas corporales de L. pictus que no fueron parasitadas, como las zonas inguinal y mandibular, por lo que es probable que no presenten un lugar favorable para los ácaros. La zona inguinal se encuentra en constante roce, lo que dificultaría la adhesión de ácaros, mientras que la zona mandibular se encuentra muy expuesta al ambiente y las escamas están muy unidas entre sí. Por el contrario, existen zonas corporales que se encuentran parasitadas exclusivamente por E. araucanensis como la zona femoral, cloacal, base de la cola, línea dorsal, abdominal, axilar y auditiva, estas 4 últimas parasitadas en baja intensidad probablemente debido a que se encuentran más expuestas al ambiente, lo que no proporciona suficiente protección contra el roce y la luz solar. Pterygosoma sp. se comporta de forma distinta, no encontrándose en las zonas anteriormente mencionadas, lo que podría deberse a la forma que posee esta especie de ácaro, que es muy similar a la forma de las escamas de la zona del flanco, donde se refugia bajo ellas protegiéndose del roce e incidencia de la luz solar (Bertrand,
2002). Ambas especies también comparten la zona gular y antehumeral; esta situación no es poco común, de hecho, es posible la coexistencia en un mismo hábitat entre especies de ácaros parásitos (Lanciani, 1970). En algunos estudios se ha registrado que la tasa reproductora de cada especie, estación del año, distribución, tipo de hábitat del hospedador y la competencia directa entre ácaros por los mejores sitios para adherirse en el cuerpo del hospedador, son factores que pueden determinar el reparto diferencial de los recursos, y esto les permite coexistir en tiempo y espacio (Lanciani, 1970; Mitchell, 1964). En este caso, Pterygosoma sp. al adherirse bajo las escamas en la zona del flanco genera un levantamiento de las escamas de L. pictus, lo que permite que queden espacios disponibles y con un mayor acceso para E. araucanensis. Además, es posible que estas zonas hayan evolucionado como una adaptación al ectoparasitismo, concentrando los ácaros en ciertas regiones corporales y minimizando el daño, debido a que estas zonas tienden a ser más elásticas y de rápida regeneración (García-de la Peña et al., 2010). Otros autores han planteado que probablemente exista una relación entre el tiempo en que el parásito se encuentra adherido sobre el hospedador y el lugar en el que se adhieren las larvas (Goldberg y Bursey, 1993), es decir, los que son ectoparásitos durante cortos periodos de tiempo se ubicarían en zonas corporales del hospedador de fácil acceso desde el ambiente. Sin embargo, los que son ectoparásitos por periodos de tiempo más largo es probable que se encuentren en zonas corporales más protegidas.

Existen múltiples factores que determinan la distribución de una especie tanto en la población de hospedadores como sobre el individuo. En este estudio no se logró evidenciar que el sexo y el tamaño corporal influyan en la distribución de $E$. araucanensis en la población de L. pictus. Existieron diferencias entre E. araucanensis y Pterygosoma sp. con respecto a la distribución corporal, lo que podría ser atribuido principalmente a la forma de las escamas que favorece a cada una de las especies en distintas zonas corporales de L. pictus.

\section{Agradecimientos}

Al laboratorio de Microevolución y Ecología de Vertebrados de la Universidad de Concepción por facilitar los ejemplares de Liolaemus pictus utilizados para la extracción de ácaros —muestreados para el proyecto FONDECYT No. 1090664-. Al Dr. Hugo Arancibia por las observaciones realizadas en el manuscrito.

\section{Referencias}

Anthony, C., Mendelson, J. y Simons, R. (1994). Differential parasitism by sex on plethodontid salamanders and histological evidence for structural damage to the nasolabial groove. American Midland Naturalist, 132, 302-307.

Arnold, E. (1986). Mite pockets of lizards, a possible means of reducing damage by ectoparasites. Biological Journal of Linnean Society, 29, 1-21.

Aubret, F., Bonnet, X., Harris, M. y Maumelat, S. (2005). Sex differences in body size and ectoparasite load in the ball python, Python regius. Journal of Herpetology, 39, 315-320

Bertrand, M. (2002). Morphologic adaptations to parasitism on reptiles: Pterygosomatidae (Prostigmata: Raphignathina). En En F. Bernini, R. Nannelli, G. Nuzzaci, y E. de Lillo (Eds.), Acarid phylogeny and evolution: adaptation in mites and ticks (pp. 233-240). Netherlands: Springer. 
Brennan, J. y Goff, L. (1977). Keys to the genera of chiggers of the Western Hemisphere (Acarina: Trombiculidae). The Journal of Parasitology, 63, 554-566.

Camacho, M. y Pérez-Lara, E. (2009). Ectoparásitos de iguana verde (Iguana iguana) y negra (Ctenosaura pectinata) en condiciones de crianza intensiva en la costa de Oaxaca, México. Ciencia y Mar, 13, 15-32.

Carothers, J. H. y Jaksic, F. M. (2001). Parasite loads and altitudinal distribution of Liolaemus lizards in the central Chilean Andes. Revista Chilena de Historia Natural, 74, 681-686.

Carvalho, A., de Araújo, A. y da Silva, H. (2006). Patterns of parasitism by Eutrombicula alfreddugesi (Oudemans) (Acari, Trombiculidae) in three species of Tropidurus wied (Squamata, Tropiduridae) from Cerrado Habitat of Central Brazil. Revista Brasileira de Zoología, 23, 1010-1015.

Clopton, R. y Gold, R. (1993). Distribution and seasonal and diurnal activity patterns of Eutrombicula alfreddugesi (Acari: Trombiculidae) in a Forest Edge Ecosystem. Journal of Medical Entomology, 30, 47-53.

Cunha-Barros, M. y Rocha, C. (1995). Parasitismo por ácaros Eutrombicula alfreddugesi (Trombiculidae) em duas espécies simpátricas de Mabuya (Sauria: Scincidae): o efeito do habitat na prevalência e intensidade parasitária. En F. Esteves (Ed.), Estrutura, funcionamento e manejo de ecossistemas brasileiros, Rio de Janeiro (pp. 307-316). Río de Janeiro: Oecologia Brasiliensis.

Cunha-Barros, M. y Rocha, C. (2000). Ectoparasitism by Chigger Mites (Eutrombicula alfreddugesi: Trombiculidae) in a restinga lizard community. Ciência e Cultura, 52, 108-114.

Cunha-Barros, M., van Sluys, M., Vrcibradic, D., Galdino, C., Hatano, F. y Rocha, C. (2003). Patterns of infestation by chigger mites in four diurnal lizard species from a resting habitat (Jurubatiba) of Southeastern Brazil. Brazilian Journal of Biology, 63, 393-399.

Delfino, M., Ribeiro, S., Furtado, I., Anjos, L. y Almeida, W. (2011). Pterygosomatidae and Trombiculidae mites infesting Tropidurus hispidus (Spix, 1825) (Tropiduridae) lizards in Northeastern Brazil. Brazilian Journal of Biology, 71, 549-555.

DeNardo, D. y Sinervo, B. (1994). Effects of steroid hormone interaction on activity and home-range size of male lizards. Hormones and Behavior, 28, 273-287.

Di Rienzo, J., Casanoves, F., Balzarini, M., Gonzalez, L., Tablada, M. y Robledo, C. (2015). InfoStat. Versión 2015. Córdoba: Grupo InfoStat, FCA, Universidad Nacional de Córdoba.

Eisen, R., Eisen, L. y Lane, R. (2001). Prevalence and adundance of Ixodes pacificus immature (Acari: Ixodidae) infesting Western fence lizards (Sceloporus occidentalis) in Northern California: temporal trends and environmental correlates. Journal of Parasitology, 87, 1301-1307.

Elgueta, E., Reid, S., Pliscoff, P., Méndez, M., Núñez, J. y Smith-Ramírez, C. (2006). Catastro de vertebrados terrestres y análisis en seis hábitats presentes en la Reserva Nacional Futaleufú, Provincia de Palena, X Región, Chile. Gayana, 70, 195-205.

Espinoza-Carniglia, M., Silva de la Fuente, M., Pérez, A., Victoriano, P. F. y Moreno, L. (2015). Fragmented host distribution and trombiculid parasitic load: Eutrombicula araucanensis and Liolaemus pictus in Chile. Acarologia, 55, 209-217.

Etheridge, R. y Espinoza, R. (2000). Taxonomy of Liolaeminae (Squamata: Iguania: Tropiduridae) and a semi-annotated bibliography. Smithsonian Herpetological Information Service, 126, 1-64.

Fajfer, M. (2012). Acari (Chelicerata)-parasites of Reptiles. Acarina, 20, $108-129$.

Fajfer, M. y González-Acuña, D. (2013). Pterygozomatid mites of a new species group ligare (Acariformes: Pterygosomatidae: Pterygosoma) parasitizing tree iguanas (Squamata: Liolaemidae: Liolaemus). Zootaxa, 3693, 301-319.

García-de la Peña, C., Contreras, A. y Castañeda, G. (2004). Infestación y distribución corporal de la nigua Eutrombicula alfreddugesi (Acari: Trombiculidae) en el lacertilio de las rocas Scleroporus couchii (Sauria: Phrynosomatidae). Acta Zoológica Mexicana, 20, 159-165.

García-de la Peña, C., Gadsden, H. y Salas-Westphal, A. (2010). Carga ectoparasitaria en la lagartija Espinosa de Yarrow (Sceloporus jarrovii) en el cañón de las piedras encimadas, Durango, México. Interciencia, 35, 772-776.

Goldberg, S. y Bursey, C. (1993). Duration of attachment of the Chigger, Eutrombicula lipovskyana (Trombiculidae) in mite pockets of Yarrow's Spiny
Lizard, Sceloporus jarrovii (Phrynosomatidae) from Arizona. Journal of Wildlife Diseases, 29, 142-144.

Grutter, A. y Poulin, R. (1998). Intraspecific and interspecific relationships between host size and the abundance of parasitic larval gnathiid isopods on coral reef fishes. Marine Ecology Progress Series, 164, 263-271.

Heredia, V., Vicente, N., Robles, C. y Halloy, M. (2014). Mites in the neotropical lizard Liolaemus pacha (Iguania: Liolaemidae): Relation to body size, sex and season. South American Journal of Herpetology, 9, 14-19.

Hoffmann, A. (1990). Los trombicúlidos de México (Acarida: Trombiculidae). México, D.F: Publicaciones Especiales del Instituto de Biología 2, Universidad Nacional Autónoma de México.

Ibargüengoytia, N. y Cussac, V. (1996). Reproductive biology of the viviparous lizard, Liolaemus pictus (Tropiduridae): biennial female reproductive cycle? Herpetological Journal, 6, 137-143.

Klukowski, M. y Nelson, C. (2001). Ectoparasite loads in free-ranging northern fence lizards, Sceloporus undulatus hyacinthinus: effects of testosterone and sex. Behavioral Ecology and Sociobiology, 49, 289-295.

Krantz, G. y Walter, D. (2009). A manual of acarology (Third Edition). Lubbock: Texas Tech University Press.

Krasnov, B., Poulin, R. y Morand, S. (2006). Patterns of macroparasite diversity in small mammals. En S. Morand, B. Krasnov, y R. Poulin (Eds.), Micromammals and macroparasites: from evolutionary ecology to management (pp. 197-231). New York: Springer.

Krasnov, B., Shenbrot, G., Khokhlova, I. y Degen, A. (2004). Flea species richness and parameters of host body, host geography and host «milieu». Journal of Animal Ecology, 73, 1121-1128.

Kubisch, E., Fernández, J. y Ibargüengoytia, N. R. (2011). Is locomotor performance optimised at preferred body temperature? A study of Liolaemus pictus argentinus from Northern Patagonia, Argentina. Journal of Thermal Biology, 36, 328-333.

Lanciani, C. (1970). Resource partitioning in species of water mite genus Eylais. Ecology, 51, 338-342.

Marler, C. A. y Moore, M. C. (1989). Time and energy costs of aggression in testosterone-implanted free-living male Mountain Spiny Lizards (Sceloporus jarrovi). Physiological Zoology, 62, 1334-1350.

Mella, J. (2005). Guía de campo reptiles de Chile: zona Central. Santiago: Ediciones del Centro de Ecología Aplicada Ltda.

Mitchell, R. (1964). A study of sympatry in the water mites genus Arrenurus (family Arrenuridae). Ecology, 45, 546-558.

Moore, S. y Wilson, K. (2002). Parasites as a viability cost of sexual selection in natural populations of mammals. Science, 297, 2015-2018.

Morand, S., Goüy de Bellocq, J., Stanko, M. y Miklisova, D. (2004). Is sexbiased ectoparasitism related to sexual size dimorphism in small mammals of Central Europe? Parasitology, 129, 505-510.

Morand, S. y Guégan, J. (2000). Distribution and abundance of parasite nematodes: ecological specialization, phylogenetic constraints or simply epidemiology? Oikos, 88, 563-573.

Oppliger, A., Giorgi, M., Conelli, A., Nembrini, M. y John-Alder, H. B. (2004). Effect of testosterone on immunocompetence, parasite load, and metabolism in the common wall lizard (Podarcis muralis). Canadian Journal of Zoology, $82,1713-1719$.

Pincheira-Donoso, D. y Núñez, H. (2005). Las especies chilenas del género Liolaemus Wiegmann, 1834 (Iguania Tropiduridae: Liolaeminae). Taxonomía, sistemática y evolución. Publicación ocasional del Museo Nacional de Historia Natural, Chile, 59, 7-486.

Poulin, R. (1996). Sexual inequalities in helminth infections: a cost of being a male? The American Naturalist, 147, 287-295.

Poulin, R. y Rohde, K. (1997). Comparing the richness of metazoan ectoparasite communities of marine fishes: controlling for host phylogeny. Oecologia, 110, 278-283.

Reiczigel, J. y Rózsa, L. (2005). Quantitative Parasitology 3.0 Budapest.

Rocha, C., Cunha-Barros, M., Menezes, V., Fontes, A., Vrcibradic, D. y van Sluys, M. (2008). Patterns of infestation by the trombiculid mite Eutrombicula alfreddugesi in four sympatric lizard species (genus Tropidurus) in Northeastern Brazil. Parasite, 15, 131-136.

Rubio, A. y Simonetti, J. (2009). Ectoparasitism by Eutrombicula alfreddugessi larvae (Acari: Trombiculidae) on Liolaemus tenuis lizard in a Chilean fragmented temperate forest. American Society of Parasitologists, 95, 244-245. 
Salkeld, D. y Schwarzkopf, L. (2005). Epizootiology of blood parasites in an Australian lizard: a mark-recapture study of a natural population. International Journal for Parasitology, 35, 11-18.

Schall, J. y Marghoob, A. (1995). Prevalence of a malarial parasite over time and space: Plasmodium mexicanum in its vertebrate host, the Western fence lizard Sceloporus occidentalis. Journal of Animal Ecology, 64, 177-185.

Schall, J., Prendeville, H. y Hanley, K. (2000). Prevalence of the tick, Ixodes pacificus, on Western fence lizards, Sceloporus occidentalis: trends by gender, size, season, site, and mite infestation. Journal of Herpetology, 34, 160-163.

Shapiro, S. y Wilk, M. (1965). An analysis of variance test for normality (complete samples). Biometrika, 52, 591-611.

Stekol'nikov, A. y González-Acuña, D. (2010). Four new species of chigger mites (Acari:Trombiculidae) of the genus Eutrombicula from Chile. International Journal of Acarology, 36, 313-325.

Stekol'nikov, A. y González-Acuña, D. (2015). A review of Chilean chiggers (Acari: Trombiculidae), with the description of a new genus and ten new species. Zootaxa, 3964, 001-043.

Tälleklint-Eisen, L. y Eisen, R. J. (1999). Abundance of ticks (Acari: Ixodidae) infesting the Western fence lizard, Sceloporus occidentalis, in relation to environmental factors. Experimental and Applied Acarology, 23, 731-740.
Tompkins, D. y Clayton, D. (1999). Host resources govern the specificity of swiftlet lice: size matters. Journal of Animal Ecology, 68, 489-500.

Tschirren, B., Fitze, P. S. y Richner, H. (2003). Sexual dimorphism in susceptibility to parasites and cell-mediated immunity in great tit nestlings. Journal of Animal Ecology, 72, 839-845.

Veloso, A. y Navarro, J. (1988). Lista sistemática y distribución geográfica de anfibios y reptiles de Chile. Bolletino del Museo Regionale Scienzia Naturali, $6,481-539$.

Vera-Escalona, I., Coronado, T., Muñoz-Mendoza, C. y Victoriano, P. (2010). Distribución histórica y actual de la lagartija Liolaemus pictus (Duméril \& Bibron, 1837) (Liolaemidae) y nuevo límite continental sur de distribución. Gayana, 74, 139-146.

Wharton, G. y Fuller, H. (1952). A manual of chiggers. Washington, D.C: Memoirs of the Entomological Society of Washington.

Wilcoxon, F. (1945). Individual comparisons by ranking methods. Biometrics, 1, 80-83.

Willson, M., Sabag, C., Figueroa, J., Armesto, J. y Caviedes, M. (1996). Seed dispersal by lizards in Chilean rainforest. Revista Chilena de Historia Natural, 69, 339-342.

Zuk, M. (1990). Reproductive strategies and disease susceptibility: an evolutionary viewpoint. Parasitology Today, 6, 231-233. 